\title{
Image Clustering and Evaluation on Impact Perforation Test by Self-Organizing Map
}

\author{
Takehiko Ogawa \\ Takushoku University \\ Japan
}

\section{Introduction}

It is important to estimate the perforation characteristics of materials in the design of the structure that collides with a flying object. Concretely, the ballistic limit velocity and the residual velocity of the material are evaluated in the impact perforation test (Backman \& Goldsmith, 1978; Zukas, 1990). Then, the evaluation from the successive images of the perforation process by a super-high-speed camera system has been studied in the mechanical or material engineering fields (Kasano, 1999; Kasano et al., 2001). In this method, a steel ball is shot into the material specimen and the perforation process is taken as successive images. Then, the characteristics of materials are estimated from the location of the steel ball and the behaviour of the specimen. However, the analysis is often difficult because of the scattered fragments. Scattering of the fragments is especially observed in acrylic composites, ceramics and their composite materials which are used in structural applications threatened by high velocity impact. As a result, the accurate evaluation of the characteristic of the material often becomes difficult.

The image clustering is necessary to evaluate the characteristic of the material from the successive images obtained by the impact perforation test. Neural networks are often used as a technique of adaptive image recognition. The neural network can be expected to classify an imperfect image by its robustness. There are a number of neural network models from the viewpoint of the learning method and the weight connection (Peincipe et al., 2000). A number of works have been reported in the pattern recognition by the multi-layered neural network (Ripley, 2007; Ogawa et al., 2006). However, an appropriate selection of learning data and appropriate parameter setting are necessary for data with large variance to use multi-layered neural networks.

The neural network model that performs unsupervised and self-organizing learning is effective to such a difficult problem (Kohonen, 1989; Kohonen, 2001). It can classify the images based on the competitive learning. The self-organizing map has been studied as an unsupervised image clustering method. Because the self-organizing map can classify the image based on the unsupervised and self-organizing learning, it is not necessary to prepare any learning data for a number of fragment patterns. We proposed to apply the selforganizing map to the image classification in the impact perforation images. Concretely, we 
classify a steel ball, background, and fragments in the impact perforation images by the selforganizing map (Horiuchi et al., 2004).

The self-organizing map performs the mapping from the input data to competitive neurons while maintaining the interrelation among the input data. The self-organizing map is suitable for the image clustering because it can express the order and the similarity between the provided data. Therefore, the distance between patterns can be estimated from the mapping result, because the self-organizing map realizes the mapping that reflects the topological relation. That is, the quantitative evaluation of the difficulty of the image clustering becomes possible. We also propose to apply the self-organizing map to evaluate the difficulty of the image clustering in the impact perforation images. Concretely, we evaluate the difficulty of the impact perforation images of each composite material by the self-organizing map (Okubo et al., 2007). Moreover, the effect of the self-organizing map is confirmed by the simulation.

In this chapter, I show the effectiveness of the self-organizing map for the image clustering of actual composite materials; polycarbonate (PC), polymethyl methacrylate (PMMA) and alumina $\left(\mathrm{Al}_{2} \mathrm{O}_{3}\right)$. Also, the difficulty of the clustering of the given image is quantitatively evaluated.

\section{Impact Perforation Test}

In the impact perforation test, a small steel ball is shot into the material specimen to examine the perforation and destruction process. The system for the impact perforation test with a super-high-speed camera is used to observe the perforation process. In this system, we can observe the states before and after the impact perforation by successive images.

When the steel ball perforates through a plate, the residual velocity of the steel ball after the perforation is expressed by several characteristics; the strength of the board, rigidity, initial form, size and so on. For example, we can estimate the material property according to the impact velocity, the residual velocity of the steel ball, and the geometric property of the material. Kasano et al. have been studying the evaluation of the material property by the impact perforation test based on the above-mentioned principle. The residual velocity $V_{R}$ is expressed by

$$
\mathrm{V}_{\mathrm{R}}=\mathrm{F}\left(\mathrm{a}_{1}, \mathrm{a}_{2} ; \mathrm{V}_{\mathrm{i}}\right)
$$

where $a_{1}, a_{2}$ and $V_{i}$ mean the material properties, geometric properties of the material and initial velocity of a steel ball. We can estimate the material property $a_{1}$, if we know the initial velocity $V_{i}$, residual velocity $V_{R}$ of the steel ball and the geometric property $a_{2}$. The perforation limit velocity is one of the important material properties, and can be estimated by the initial velocity and the residual velocity that are obtained in the impact perforation test (Kasano et al., 2001).

As one of the measuring method of the velocity of a steel ball, a super high-speed camera is used. In this system, the steel ball launched from the shooting device perforates through a monotonous material plate in the high temperature furnace, and the appearance is taken of a picture with the high-speed camera. The high-speed camera of this system can take a picture of four successive images. The experimental setup that we used is shown in Fig.1. We can measure the velocity of the steel ball with the high-speed camera from the 
successive images of the perforation. However, the location of the steel ball cannot often be measured in precision by the fragments of the destructed material in the perforation image of an actual material. In the current system, the image of the impact perforation test is visually classified. The classification from the successive images is often difficult because of the fragments of the material. Therefore, precise image clustering of a steel ball, background and fragments is necessary. We propose the use of neural network to classify them in the image with degradation by fragments of the specimen. We show the image classification of the images degraded by the scattered material fragments accurately by using the neural network (Ogawa et al., 2003).

The successive images of the impact perforation test with actual composite materials; polycarbonate $(\mathrm{PC})$, polymethyl methacrylate (PMMA) and alumina $\left(\mathrm{Al}_{2} \mathrm{O}_{3}\right)$ specimens are shown in Fig.2. The number drawn in the figure shows the photographed order. The image of PC specimen is so clear that we can visually classify it, sufficiently. However, the classification of the image of PMMA specimen is a little more difficult than that of PC. Moreover, the classification of the image of alumina is too difficult to classify because of scattered fragments. The aim of this study is to classify these images accurately. The plate size, thickness of the specimen, the impact velocity of the steel ball and the interframe time of successive images are shown in Table 1 . The size of the steel ball was $5 \mathrm{~mm}$ in diameter, and $0.5 \mathrm{~g}$ in mass.

The polycarbonate (PC) has the transparency equal with the glass and the highest impactproof in plastic, and is used for the consumer electronic and the mobile device and so on. The impact-proof of PC equals a metallic material. However, there is a fault of weakness to an alkaline medicine and an organic solvent. The polymethyl methacrylate (PMMA) has the highest scratch-proof and transparency in transparent plastic, and is used for the window, lens and the housing of various equipments. However, it is a little inferior to the impactproof, and is a polymeric material that causes the same brittleness destruction as ceramics. Because the alumina is light and has the corrosion-proof, it is expected as a material of the machine structure in the ultra high temperature. However, there is a fault that resistance to the mechanical shock and thermal shock is extremely low because the destruction toughness is low and fragile. The impact perforation images of these three kinds of specimens are classified into the steel ball, the background, and the fragments (material).

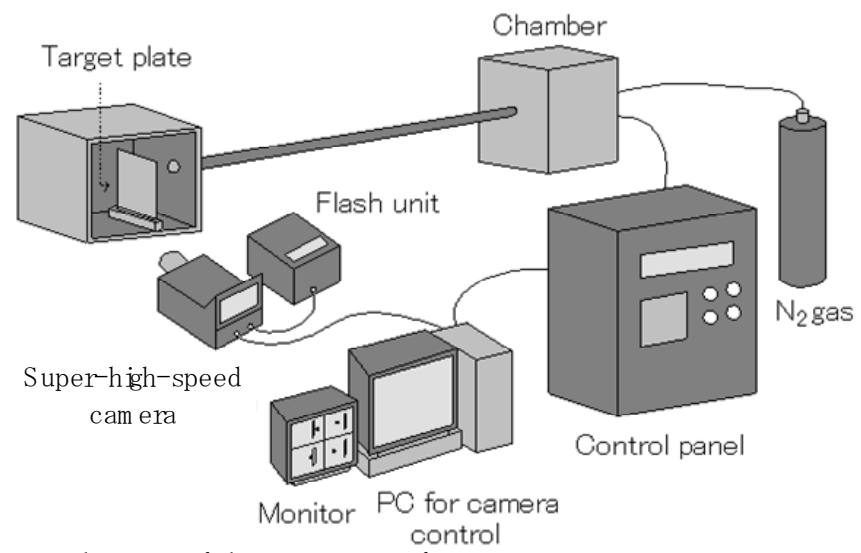

Fig. 1. Experimental setup of the impact perforation test. 


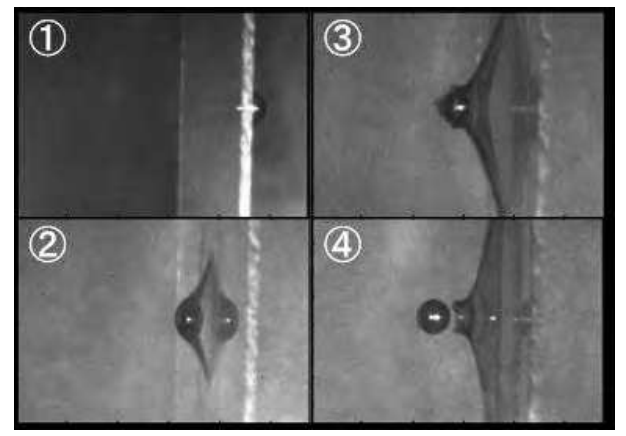

(a)

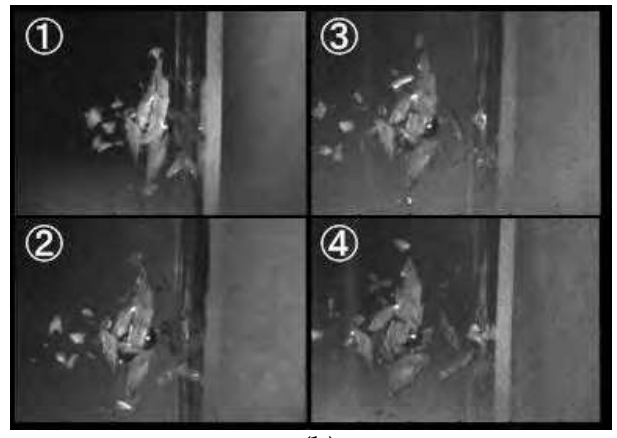

(b)

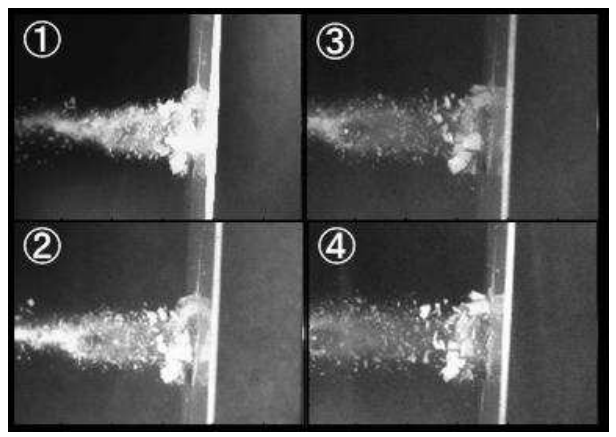

(c)

Fig. 2. Successive images obtained by super high-speed camera in the impact perforation test with composite materials, (a) polycarbonate (PC) specimen, (b) polymethyl methacrylate (PMMA) specimen and (c) alumina specimen.

\begin{tabular}{|c|c|c|c|}
\hline Material & PC & PMMA & alumina \\
\hline Image size $($ pixel) & $1172 \times 770$ & $1000 \times 656$ & $1172 \times 770$ \\
\hline Specimen's size $(\mathrm{mm})$ & $100 \times 100$ & $80 \times 60$ & $80 \times 80$ \\
\hline Specimen's thickness $(\mathrm{mm})$ & 1.5 & 3.0 & 1.5 \\
\hline Initial velocities of a steel ball $(\mathrm{m} / \mathrm{s})$ & 220 & 309 & 224.6 \\
\hline Interval time of successive images $(\mu \mathrm{s})$ & 50 & 150 & 50 \\
\hline
\end{tabular}

Table 1. Parameters of the impact perforation test and images.

\section{Self-Organizing Map}

There are a number of supervised learning models and unsupervised learning models in the neural network. Typical examples of the former and the latter are the multi-layered neural network and the self-organizing map, respectively. The multi-layered neural network learns the input-output relation using the error back-propagation method. Because the number of learning data are limited when the input-output mapping is learned by the multi-layered neural network, the network supplements the answer of the problem which was not provided as the learning data with the generalization ability. The multi-layered neural 
network has the problem of over-learning in which the learning progresses only for the provided learning data and the generalization ability decreases (Ogawa, 1992).

On the other hand, the self-organizing map is an unsupervised learning model of neural network, which is based on the competitive learning method. Typical self-organizing map is composed of an input layer, a competitive layer and interlayer connection weights, as shown in Fig.3. An input neuron has the connection weights with all competitive neurons. Since the learning expresses the interrelation of input data, a competitive layer forms the mapping that reflects the distributions of input data. In the self-organizing map, we need not provide to which category input data belongs, and input data are classified by selforganizing. The interrelation among the input data is mapped to the neurons arranged in one or two-dimensional area. Therefore, it is considered that the problem of supervized learning model can be overcome.

At the learning phase of the network, the neuron that responds most strongly becomes a winner while a lot of neurons respond for a given input, and the connection weights of the winner neuron and the neighborhood neurons are updated. The learning is done for not only the winner of the competition but also their neighborhoods. In a word, the adjacent neurons have mutually similar connection weights because they learn the similar data. The template that reflects a statistical distribution of data and mutual analogous relationship is formed by such learning. Therefore, the similarity of the input data can be measured according to the relative distance of them. The concrete procedure of competitive learning of the self-organizing map is as follows. First, it identifies a winner neuron. Then, the weights of the winner neuron and the neighborhoods are updated by the input vector in each learning step. The weights of the winner neuron and neighborhood are updated in proportion to the learning rate. The self-organizing map is suitable for clustering that classifies the data set into some groups without learning data, because it is able to express the similarity of the data set.

In this study we propose to use the self-organizing map for classifying the impact perforation images. The self-organizing map is used to distinguish a steel ball, background and fragments. In the impact perforation images, the shapes of the fragments are different on each material, experimental condition and so on. The image in a steel ball is also slightly different according to differences of the quantity of light and so on. So, it is difficult to prepare the appropriate and sufficient learning data for the material specimen and the experiment environment, beforehand. We use the self-organizing map which is one of the competitive learning type neural networks. As mentioned above, the unsupervised learning of the self-organizing map is not affected by the quality of the learning data. The impact perforation image is classified by the self-organizing map. The self-organizing map recognizes the category by a partial space which the data belongs to the division of the category of the feature vector space, where data exists with the represented template. 


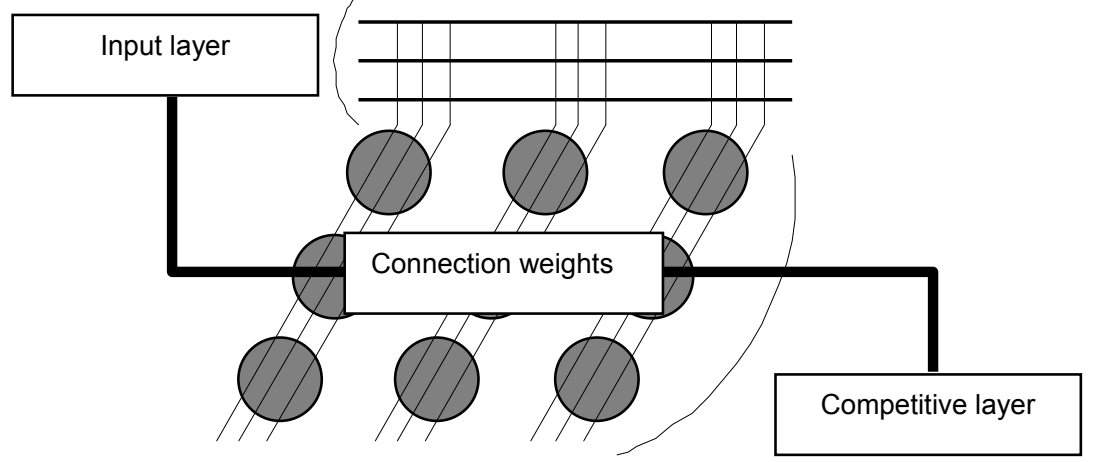

Fig. 3. Typical architecture of self-organizing map.

\subsection{Unsupervised Learning of Self-Organizing Map}

In the self-organizing map, a number of neurons fire to the given input. The neuron with the strongest output becomes a winner, and the connection weights are updated to the winner neuron and its neighbourhoods. The input neurons and the competitive neurons are connected in parallel between all neurons through the connection weights. In general, these weights have different values for different neurons. The output values of the competitive neuron to a certain input vector are compared by all competitive neurons, and the position that reaches the highest value in a certain measure is considered to be the response position. Here, we consider the network with $\mathrm{M}$ input neurons and $\mathrm{N}$ competitive neurons. The connection weights vector from the $\mathrm{i}$-th input neuron and a certain input vector are assumed to be $\mathrm{w}_{\mathrm{i}}=\left(\mathrm{w}_{\mathrm{i} 1}, \mathrm{w}_{\mathrm{i} 2}, \ldots, \mathrm{w}_{\mathrm{iN}}\right), \mathrm{i}=1,2, \ldots, \mathrm{M}$ and $\mathrm{x}=\left(\mathrm{x}_{1}, \mathrm{x}_{2}, \ldots, \mathrm{x}_{\mathrm{M}}\right)$. When input data are given, we compare the distances between the input vector and each competitive neuron to dicide the winner neuron $k$. The distance $D_{j}$ on $j$-th competitive neuron is defined by euclid distance as

$$
D_{j}=\sum_{i=1}^{M}\left|x_{i}-w_{i j}\right|
$$

where $M$ means the number of input neurons. Based on the competitive learning, the output of the neuron $Z_{k}$ with the most similar weight becomes one, while the output of the other neuron become zero. Then, the weights of the winner neuron and its neighborhoods $N_{k}$ are updated. The weights of the winner neuron from the i-th input neuron are updated as

$$
\mathrm{w}_{\mathrm{ik}}(\mathrm{t}+1)=\mathrm{w}_{\mathrm{ik}}(\mathrm{t})+\alpha(\mathrm{t})\left(\mathrm{x}_{\mathrm{i}}-\mathrm{w}_{\mathrm{ik}}(\mathrm{t})\right)
$$

where $\alpha(t)$ means the learning coefficient that is reduced as the learning epoch. In general, the weights of the neighborhood neuron is corrected at the update rate that is lower than that of the winner neuron. In this study, the weights of the neighborhood neurons are updated as

$$
\mathrm{w}_{\mathrm{ik}}(\mathrm{t}+1)=\mathrm{w}_{\mathrm{ik}}(\mathrm{t})+\frac{\alpha(\mathrm{t})}{2}\left(\mathrm{x}_{\mathrm{i}}-\mathrm{w}_{\mathrm{ik}}(\mathrm{t})\right)
$$


The other neurons are not corrected as

$$
\mathrm{w}_{\mathrm{ik}}(\mathrm{t}+1)=\mathrm{w}_{\mathrm{ik}}(\mathrm{t})
$$

The range of the correction of the weights is shown like Fig.4. The weight $\mathrm{w}_{\mathrm{ik}}$ of the winner neuron $\mathrm{k}$ is corrected in the direction of the input vector $\mathrm{x}_{\mathrm{i}}$, and approaches to the input vector $x_{i}$. Parameter $\alpha(t)$ is a learning coefficient, and is the parameter that adjusts how to approach it to $x_{i}$. Variable $t$ means the epoch number, and the set $\mathrm{N}_{\mathrm{c}}(\mathrm{t})$ of the neighborhood neurons of winner $k$ and the learning coeffiecinet $\alpha(t)$ are the function of $t$. Learning coefficient $\alpha(t)$ is initially set to the comparatively large value, and is decreased gradually with the learning epoch $t$. Also, the size of the neighborhood region $N_{c}(t)$ is decreased with the learning epoch. All the weights of the neuron move greatly toward the input area where the input vector exists. Then, the map orders itself to the given input vector, as the neighborhood distance decreases. The relation between the weight correction and the learning coefficient is shown in Fig.5.

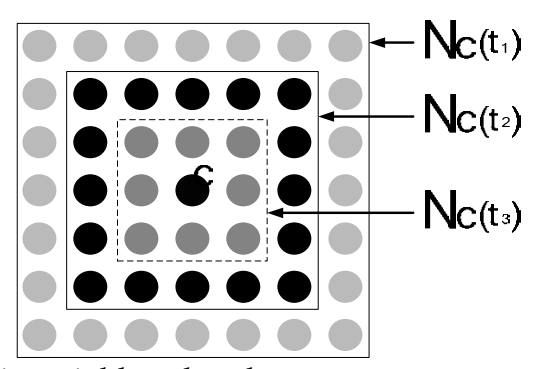

Fig. 4. Winner neuron and its neighbourhood neurons.

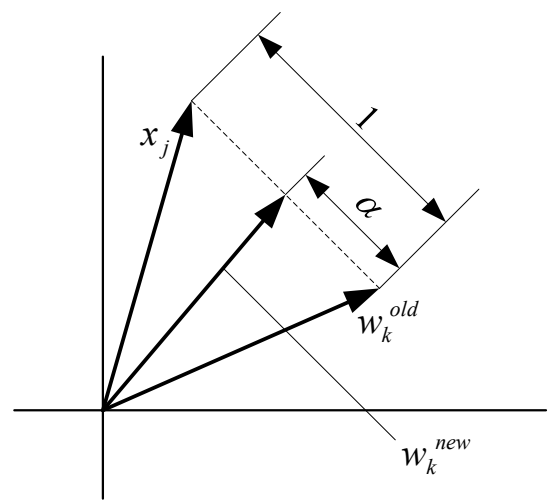

(a)

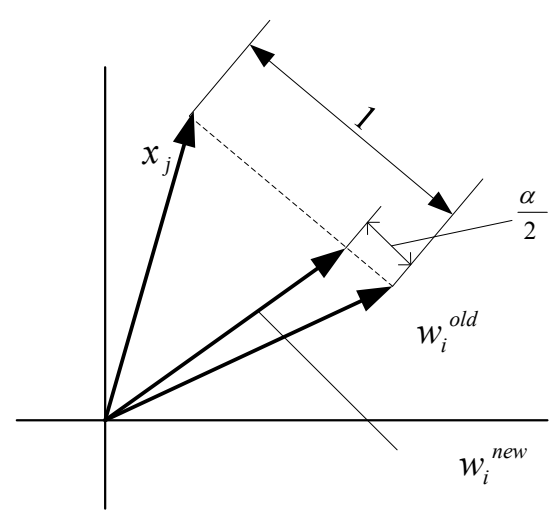

(b)

Fig. 5. Relation between weight correction and learning coefficient, (a) winner neuron (b) neighborhood neurons. 


\section{Image Clustering and Evaluation by Self-Organizing Map}

In this section, the architecture and the procedure of the self-organizing map for image clustering and evaluation on the impact perforation test are explained. The self-organizing map used in this study has the one-dimensional competitive layer. The input is the onedimensional vector by which the feature of the image is extracted, and the output is a mapped vector which appears on the one-dimensional competitive layer. The purpose of the image clustering is the classification of a steel ball, background and fragments in the impact perforation images. Also, the purpose of the image evaluation is to evaluate the difficulty of the image clustering from the output distribution on the competitive layer. The network architecture used in this study is shown in Fig.6.

The network is used in the three steps; the learning, the classification, and the evaluation. They are explained by the following subsections.

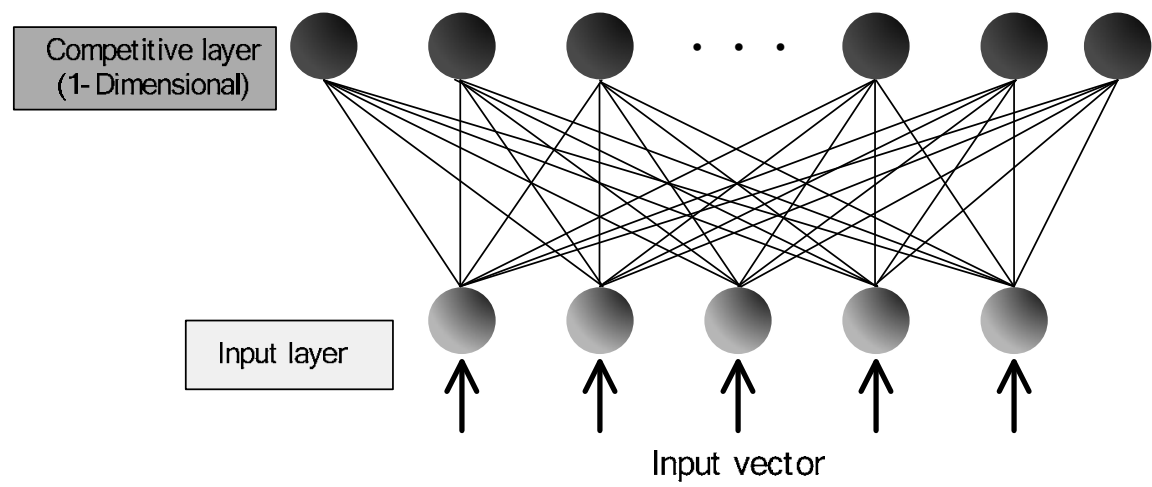

Fig. 6. Network architecture

\subsection{Learning by Self-Organizing Map}

Here, the learning method of the self-organizing map is explained. In general, we compose the input vector after compressing the image, and classify it in the image classification. In this study, the sub-images of the steel ball, the background, and the fragments are extracted from the original images. We use two sub-images of specimen fragments, because the fragments of the specimen is various shape. The quantitative features are calculated from those sub-images as input vectors. The mapping forms the template of the steel ball, the background, and the fragments of the specimen by unsupervised learning of the input vector obtained from these learning sub-images.

The network first identifies the winner neuron for the given input vector. Then, the vector expressed by the weights of the winner neuron and those of the neighborhood neurons are brought close to the value of the input vector in each learning step. The weights of the winner neuron are changed for proportion to the learning rate, while those of the neighborhood neuron are changed for proportion to the half of the learning rate. The learning rate and the neighborhood distance of the winner neuron are updated through two phases of the ordering phase and the tuning phase. To use these two phases, the following four are defined (Demuth et al., 2009). 


\section{Ordering phase}

Ordering phase lasts for the given number of steps. The neighborhood distance starts at a given initial distance, and decreases to the tuning neighborhood distance. As the neighborhood distance decreases over this phase, the neurons of the network typically order themselves in the input space with the same topology in which they are ordered physically.

\section{Tuning phase}

Tuning phase lasts for the rest of learning or adaptation. The neighborhood distance stays at the tuning neighborhood distance, which should include only close neighbors, i.e., typically 1.0. The small neighborhood fine-tunes the network, while keeping the ordering learned in the previous phase stable.

As with competitive layers, the neurons of a self-organizing map will order themselves with approximately equal distances between them if input vectors appear with even probability throughout a section of the input space. If input vectors occur with varying frequency throughout the input space, the feature map layer tends to allocate neurons to an area in proportion to the frequency of input vectors there. Thus, feature maps, while learning to categorize their input, also learn both the topology and distribution of their input. The summary of the procedure is as follows.

Step 1. The sub-images of the steel ball, the background, and fragments of specimen are extracted from the impact perforation images as a learning image.

Step 2. The input vector by quantitative features is calculated from the extracted subimages.

Step 3. The calculated input vector is input to the network to update the weights.

\subsection{Image Clustering by Self-Organizing Map}

Image clustering by the self-organizing map is performed by the learned network. The learning images are mapped to the competitive layer. While the output of only the nearest neuron in the map becomes 1.0 if the input data of the image classified into the network is provided, those of other neurons become 0.0 . The procedure of clustering is as follows.

Step 1. The labeling is done to the neuron in the competitive layer after the learning.

Step 2. The images to be classified is divided into the decided size, and the input vector is calculated.

Step 3. After the calculated input vector is input to the network, and the network outputs it from the result of the response and the labeling in the competitive layer.

To classify the input image, it is necessary to confirm which neuron in the competitive layer represents the input data of the learning image. We input each learning image and label the responding competitive neurons. As a result, the labeling is done to the neuron in the competitive layer and the image is classified. Figure 7 shows the concept of the labeling. The concept of the network architecture in the image clustering is shown in Figure 8.

The classification images are four successive images, and the classication is performed to each image. The classification images are scanned and cut out to the sub-images. After the calculation of the input vector for each sub-image, it is classified with the label of the neuron that responds to the input vector. 


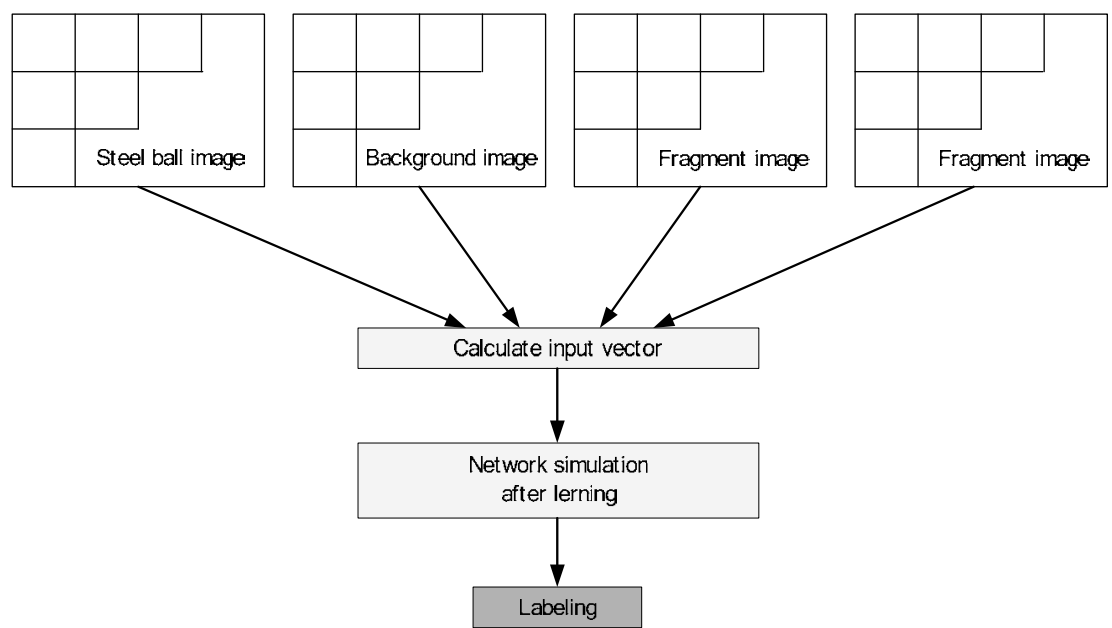

Fig. 7. Concept of labeling

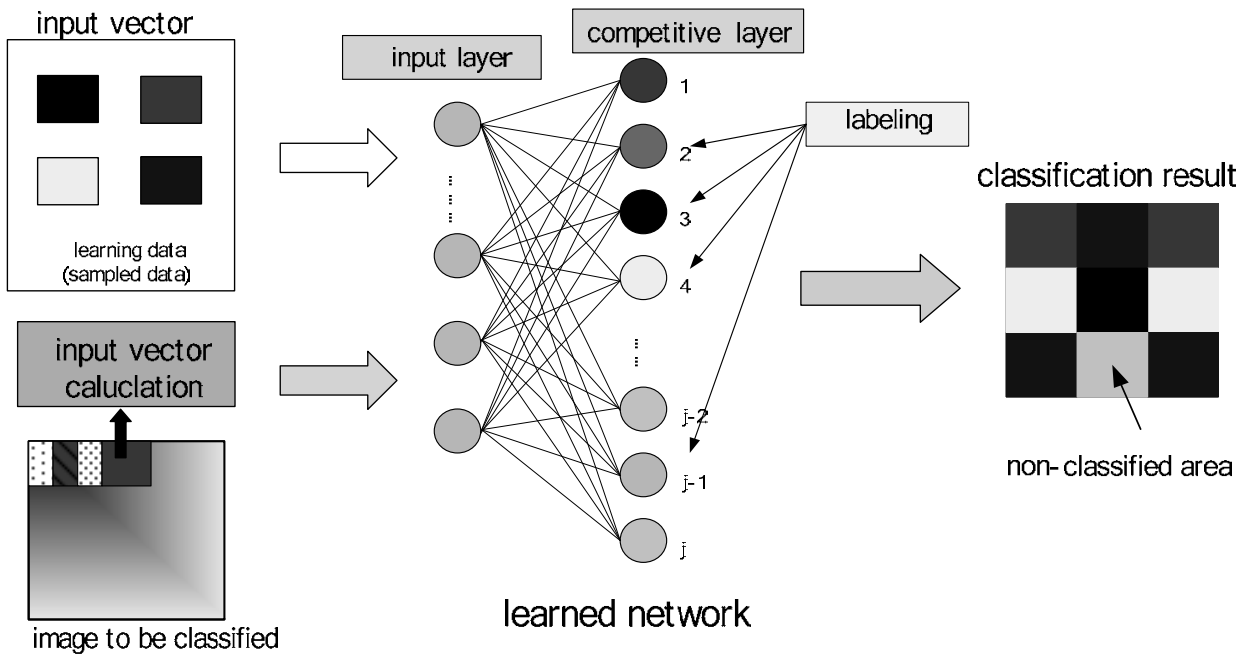

Fig. 8. Concept of the network architecture in image clustering

\subsection{Image Evaluation by Self-Organizing Map}

The self-organizing map is able to map the input to the competitive layer with the topological relationship between the given input vectors. In a word, the topological relationship can be estimated from the distribution expressed on the competitive layer neuron. Based on this feature, we proposes the method of quantitatively evaluating the difficulty of the classification of the given image by using the self-organizing map for the topological relationship between images of the steel ball, the background, and the fragments of specimen. 
The steel ball, the background, and the fragments are labeled at the same time as the image's being classified by the self-organizing map. The location where each neuron responds to the output distribution on the competitive layer can be shown from the result of the labeling. Then, the location of fired neuron on the competitive layer in each pattern is confirmed. Figure 9 shows the concept of the image evaluation.

The difficulty and accuracy of the image classification can be estimated from the location of the firing neuron on the competitive layer for each pattern. For instance, if each pattern of the steel ball, the background, and fragments is classified by the firing neuron position of a competitive layer in each pattern clearly, the image can be judged to be an easy classification. However, it can be judged that the image is difficult classification if the firing neuron position by each pattern is overlapping. Also, it is similarly used as an evaluation method of the classification result. If each pattern is separate, the image is classified almost accurately. On the other hand, if each pattern overlaps, it is appreciable with an inaccurate image classification.

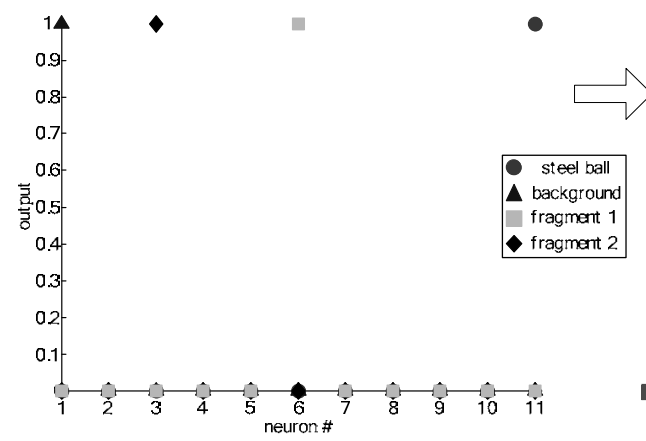

(a)

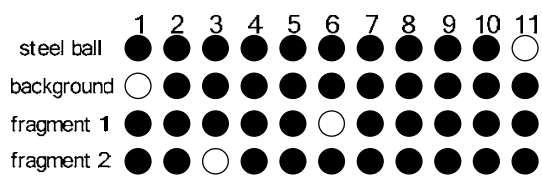

(b)

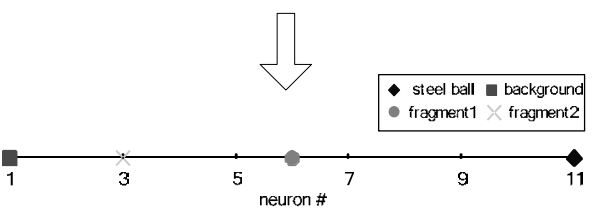

(c)

Fig. 9. Concept of image evaluation by self-organizing map, (a) result of labeling, (b) situation of firing on competitive layer, and (c) location of firing neuron on competitive layer for each pattern.

\subsection{Input Vector of Self-Organizing Map}

In this study, two kinds of input vector are examined to classify the image. The first method is to calculate the quantitative features from the sub-image and to compose the input vector. First of all, the sub-image is extracted from the impact perforation image. Then, the quantitative features; the standard deviation, the difference of the maximum value and minimum value, the median value, the maximum frequency value, the mean values of the four corners, the maximum values, the minimum value and the mean value in the pixels. The one used for the input vector calculation was selected from these quantitative features by the trial and error.

Next, the method of inputting immediate pixel values is examined. The sub-image is extracted from the impact perforation image as well as the input vector by the amount of characteristic. Then, the pixel values of five points are extracted from the sub-image. The input vectors used in the simulation are shown in Table 2. 


\begin{tabular}{|c|c|c|}
\hline Input vector & Quantitative features & Pixel values \\
\hline PC & $\begin{array}{c}\text { Standard deviation } \\
\text { Difference of max and min values } \\
\text { Maximum value } \\
\text { Minimum value }\end{array}$ & \\
\hline PMMA & $\begin{array}{r}\text { Difference of max and min values } \\
\text { Median value }\end{array}$ & \multirow{3}{*}{ Five pixel values } \\
& Maximum frequency value & \\
& Maximum value & \\
& Minimum value & \\
& Mean value & \\
& Standard deviation & \\
& Difference of max and min values & \\
& Median value & \\
Alumina & Maximum frequency value & \\
& Maximum value & \\
& Minimum value & \\
& Mean value & \\
& &
\end{tabular}

Table 2. Input vectors used in the simulation.

\section{Simulation}

In this study, we use the impact perforation images of PC (polycarbonate), PMMA (polymethyl methacrylate) and alumina, shown in Fig.2. Each image consists of four successive photos. The parameters are shown in Table 1. The PC image can be visually classified. The classification of the PMMA image is comparatively easy though it is not clearer than the PC image. However, it is difficult to classify the alumina image because of the scattering of the fragments. In this study, these images are classified by the image recognition network using the self-organizing map. In addition, the quantitative evaluation of the difficulty of image classification by the self-organizing map is examined.

The learning image and the classification image are extracted from the original impact perforation images. As for the learning image of PC, the steel ball, the background and two material images of 16x16 pixels are extracted from the second image. The learning image of PMMA is extracted from the third image in the same size as PC. As for the learning image of the alumina image, the steel ball, the background of 16x16 pixels and two fragment images of $48 \times 48$ pixels are extracted from the third image. These learning images of PC, PMMA and alumina are shown in Figs. 10, 11 and 12 respectively. Then, the classification images are extracted from each original image (PMMA, PC and alumina). The extracted images are shown in Fig. 11. The size of each classification image is $256 \times 256$. These four images are classified into four kinds; steel ball, background and two fragments.

To examine two kinds of input values in which the quantitative features and the immediate pixel values of the learning image, we input these to the network in learning phase. The classification simulation is carried out by two methods of the quantitative features and the immediate pixel values, and the difficulty of the image and the accuracy of the classification are evaluated from the output distribution of the competitive layer. 
The network parameters are shown in Table 3. The network parameters are changed according to the input vector and the classified image. In the case of the quantitative features, we decided to adopt the features by trial and error. On the other hand, we sampled five pixels as the immediate pixel value input. We used 11 neurons that were arranged in one dimension as a competitive layer.

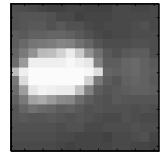

(a)

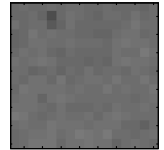

(b)

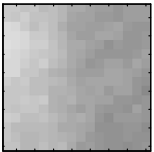

(c)

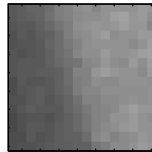

(d)

Fig.10 Learning image of PC, (a)steel ball, (b)background, (c) fragment1, and (d)fragment2

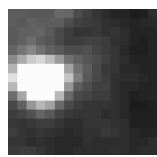

(a)

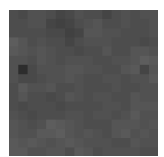

(b)

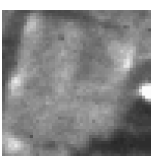

(c)

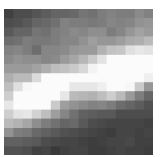

(d)

Fig. 11. Learning image of PMMA, (a)steel ball, (b)background, (c) fragment1, and (d)fragment2

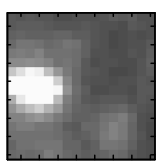

(a)

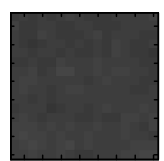

(b)

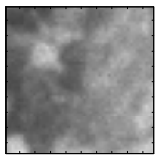

(c)

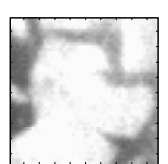

(d)

Fig. 12. Learning image of alumina, (a)steel ball, (b)background, (c) fragment1, and (d)fragment2

\begin{tabular}{|c|c|c|c|}
\hline Material & PC & PMMA & alumina \\
\hline Input vector & $\begin{array}{c}4 \text { features } \\
\text { or 5 pixel values }\end{array}$ & $\begin{array}{c}6 \text { features } \\
\text { or 5 pixel values }\end{array}$ & $\begin{array}{c}7 \text { features } \\
\text { or 5 pixel values }\end{array}$ \\
\hline Number of input neurons & 4 or 5 & 6 or 5 & 7 or 5 \\
\hline Number of competitive neurons & \multicolumn{3}{|c|}{0.9} \\
\hline Ordering phase step number & \multicolumn{3}{|c|}{0.01} \\
\hline Ordering phase learning rate & \multicolumn{3}{|c|}{1000} \\
\hline Tuning phase step number & \multicolumn{3}{|c}{500} \\
\hline $\begin{array}{c}\text { Tuning phase neighbourhood } \\
\text { distance }\end{array}$ & \multicolumn{3}{|c}{} \\
\hline Learning epoch number & \multicolumn{3}{|c|}{} \\
\hline
\end{tabular}

Table 3. Network parameters. 

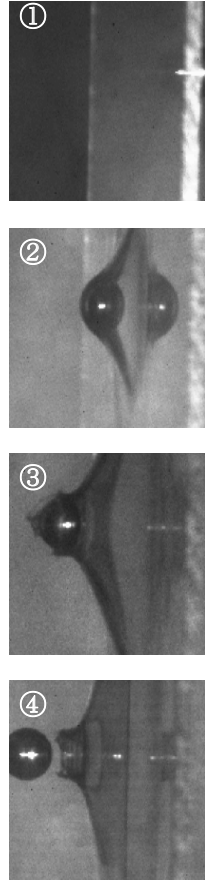

(a)
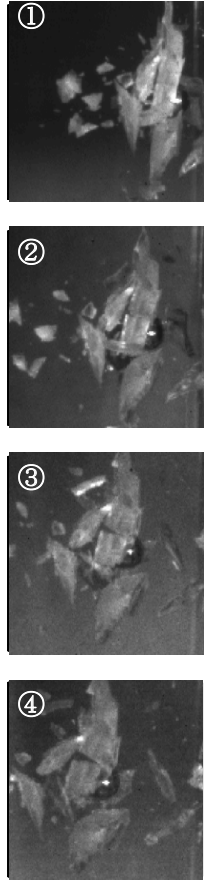

(b)
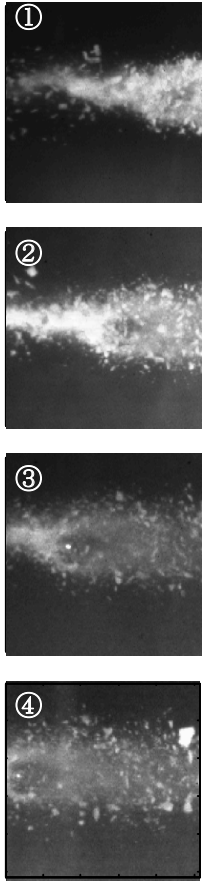

(c)

Fig. 13. Classification images, (a) PC, (b) PMMA and (c) alumina

\subsection{Results}

The simulation was carried out to confirm the classification and the quantitive evaluation of the difficulty of the classification by the amount of characteristic and pixel value input of PC, PMMA and alumina impact perforation image.

First, the classification result of PC is shown in Fig. 14. The white area in the classification result shows the steel ball. The gray area shows the background and fragments. A black area means unclassificated area. According to the result, the place of the steel ball can be distinguished. Neither the background nor the material are distinguished accurately partially. The output distribution of a competitive layer for each patterns is shown in Fig.15. In Fig.15, the white circle and the black circle mean the acted and not acted neurons, respectively. From result of Fig.15, it is understood that each pattern is separate to each learning image clearly in the PC image. Because the distance between patterns of the steel ball and the material is considerably large, it is considered that the distinction is the easiest.

Next, the classification result of PMMA is shown in Fig.16. The result is shown similar to the case of PC. From the result, we can see to distinguish the steel ball clearly. Moreover, because the distinction of the background and the fragment is easy, it is possible to classify it. The output distribution of a competitive layer is shown in Fig.17. As for the PMMA image, each pattern is separate to each learning image clearly. Because the distance between a steel ball and fragment patterns is large, it is considered that the distinction becomes easy. 
Finally, the classification result of alumina is shown in Fig.18. From the result, it is difficult to distinguish the steel ball because of the scattered fragments. However, the fragments and the background is distinguished correctly. The output distribution of a competitive layer is shown in Fig.19. In the alumina image, it is considered that each pattern adjoins each learning image. Because the distance between a steel ball and fragment patterns is small, it is considered that the distinction becomes difficult.

According to the above-mentioned result, the classification of the steel ball that is necessary to estimate the strength property of the material was sufficiently performed in three impact perforation images. Also, the classification of the fragments that is important to estimate the destruction property was almost done, too. Moreover, it was shown that the distinction of the steel ball was easy in order of the PC image, the PMMA image, and the alumina image according to the result of the evaluation by the output distribution in a competitive layer. From the results of Figs. 15, 17 and 19, the mean distances between the firing positions to the steel ball and the fragments on the competitive layer are calculated and are shown in Table 4 . These values are almost corresponding to an intuitive difficulty to classify each image. That is, it was clarified that the output distribution of the competitive layer expressed the difficulty of the quantitative evaluation of the classification difficulty by the simulation. Especially, it was confirmed that the distance between the competitive layer neurons that responded to the input vector of the steel ball and the fragments expressed the classification difficulty. Therefore, the difficulty of the image classification can be quantitively evaluated by measuring the positions of the responding competitive layer neurons.
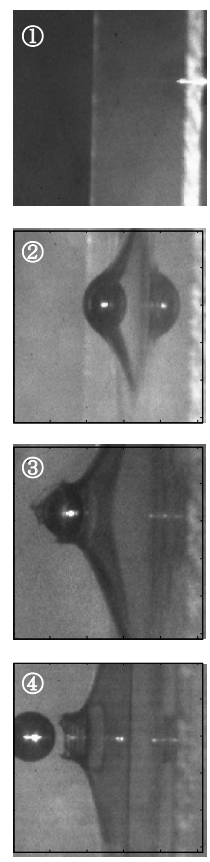

(a)
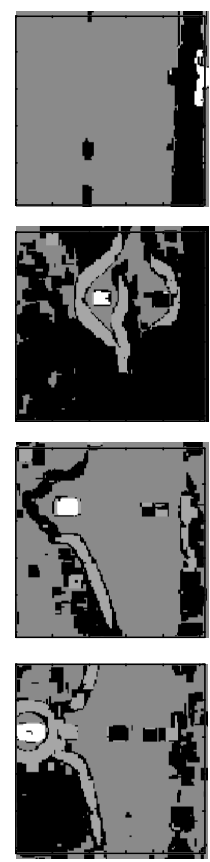

(b)
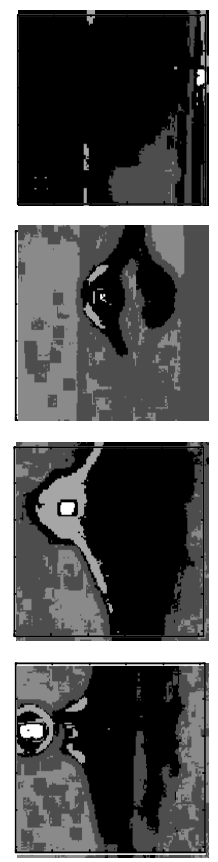

(c)

Fig. 14. Classification result for PC specimen image. (a) original image, (b) result for quantitative feature input, (c) result for pixel value input. 


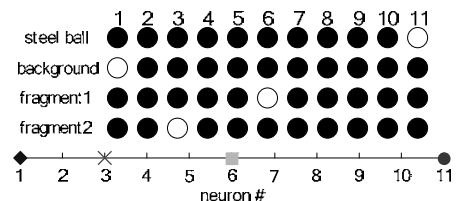

(a)

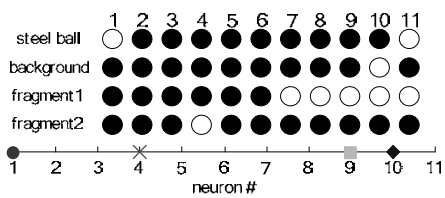

(b)

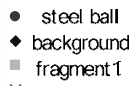

fragment1
$\times$ fragment2

Fig. 15 Output distribution of a competitive layer for PC specimen image. (a) result for quantitative feature input and (b) result for pixel value input.
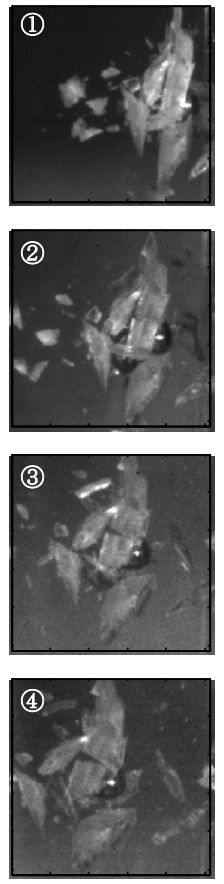

(a)
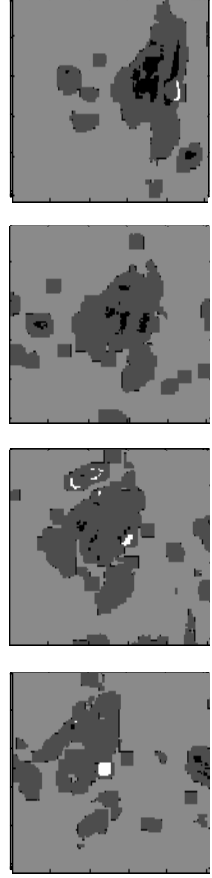

(b)
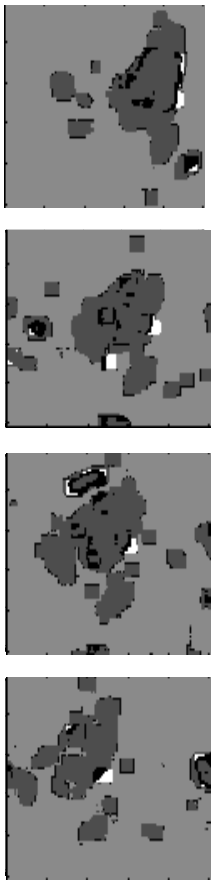

(c)

Fig. 16. Classification result for PMMA specimen image. (a) original image, (b) result for quantitative feature input, (c) result for pixel value input.

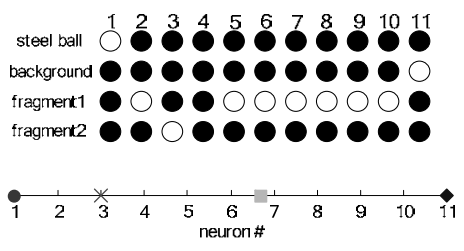

(a)
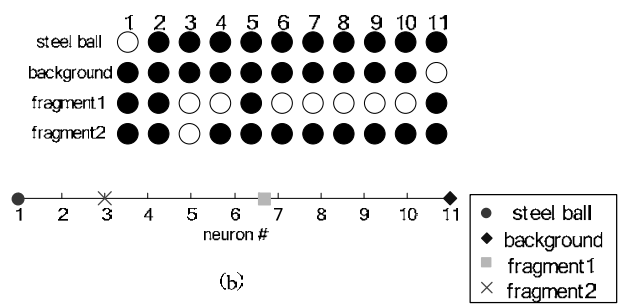

Fig. 17. Output distribution of a competitive layer for PMMA specimen image. (a) result for quantitative feature input and (b) result for pixel value input. 

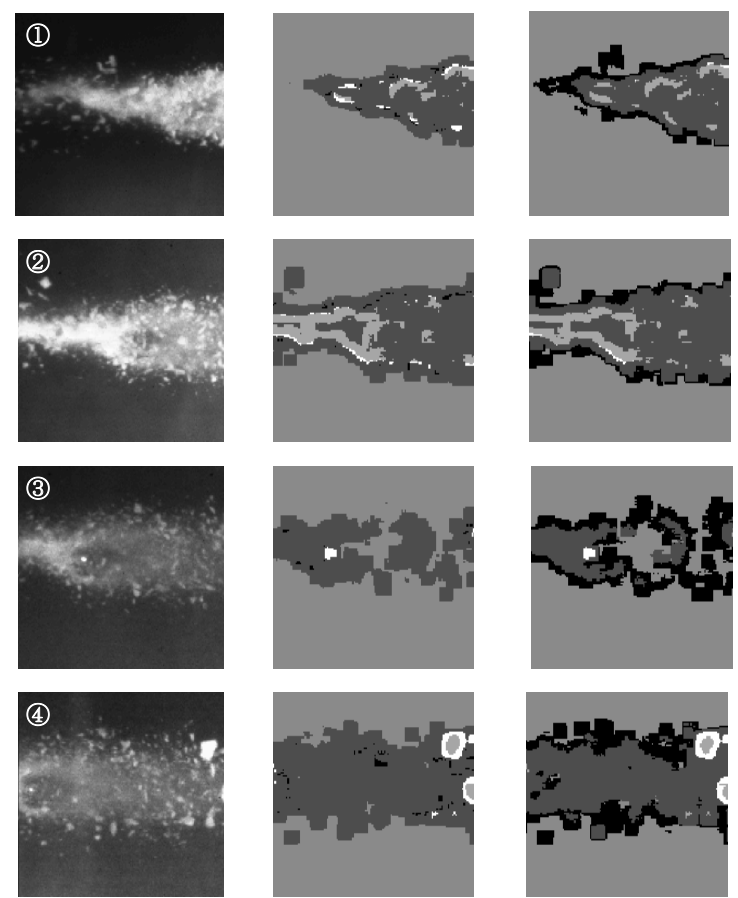

Fig. 18. Classification result for alumina specimen image. (a) original image, (b) result for quantitative feature input, (c) result for pixel value input.

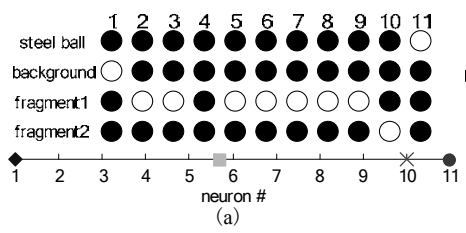

(a)

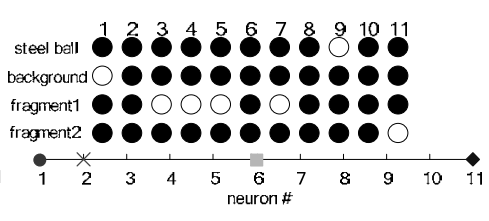

(b)

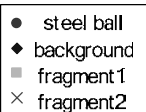

Fig. 19. Output distribution of a competitive layer for alumina specimen image. (a) result for quantitative feature input and (b) result for pixel value input.

\begin{tabular}{|c|c|c|c|}
\hline Mnput Materials & PC & PMMA & alumina \\
\hline Quantitative features & 6.5 & 3.6 & 3.1 \\
\hline Pixel values & 5.5 & 3.9 & 3.1 \\
\hline
\end{tabular}

Table 4. Distances between firing neurons corresponding to a steel ball and fragments.

\section{Conclusion}

In this study, we proposed to use the self-organizing map for the classification of the impact perforation image, as one of the image recognition problems by the neural network. Then, the classifications of three impact perforation images (PC, PMMA and alumina specimens) 
were used to examine the image recognition by the self-organizing map. The quantitative features and the immediate pixel values of the images were used and simulated as the network inputs, and the effect of the self-organizing map was confirmed. Since the selforganizing map maintained the topological order in the data space, it was confirmed to be able to judge the difficulty of the image classification from the output result.

As for the PC image, the steel ball was clearly distinguished but the material was not classified sufficiently. The reason is considered that the self-organizing map was not able to extract the feature of the image accurately. In the PMMA image, the steel ball and the fragments were classified clearly. The reason is considered that the self-organizing map extracted the features of the image almost accurately. In the alumina image, the steel ball was not classified sufficiently, because of the scattered fragments. However, the fragments and the background were separated clearly. Moreover, the difficulty of the image classification was evaluated from the output distribution on the competitive layer neuron to each pattern. Concretely, the reason why the classification of the steel ball in the alumina image is difficult was shown though that in the PC and PMMA images was easy.

As the future problems, the improvement of the classification accuracy and the evaluation accuracy is considered. First of all, we have to examine the input vector calculation method to extract more features for more accurate classification. It is necessary to examine the vector calculation method that extracts the features of the image, while the two types of the input vector; the quantitative features and the immediate pixel values were used in this study. Moreover, it is necessary to examine the relation between the accuracy and the composition of the competitive layer for the quantitative evaluation of the difficulty of the image classification. It is considered that there is an influence on the classification accuracy by increasing the number of competitive layer neurons to improve the evaluation accuracy.

\section{References}

Backman, M. E. \& Goldsmith, W. (1978). The Mechanics of Penetration of Projectiles into Targets, International Journal of Engineering Science, vol. 16, issue 1, pp.1-99.

Demuth, H.; Beale, M. \& Hagan, M. (2009). Neural Network ToolboxTM 6 User's Guide, The MathWorks, Inc.

Horiuchi, T.; Ogawa, T. \& Kanada, H. (2004). Analysis of Impact Perforation Images Using Self-Organizing Map, Proceeding of the SICE Annual Conference, pp.605-608.

Kasano, H. (1999). Recent Advances in High-Velocity Impact Perforation of Fibber Composite Laminates, JSME International Journal A, Vol. 42-2, pp.147-157.

Kasano, H. ; Okubo, T. \& Hasegawa, O. (2001). Impact Perforation Characteristics of Carbon/Carbon Composite Laminates, International Journal of Materials and Product Technology, Vol.16, No.1-3, pp.165-170.

Kohonen, T. (1989). Self-Organization and Associative Memory, Springer-Verlag, ISBN: 9783540183143, Berlin.

Kohonen, T. (2001). Self-Organizing Maps, Springer-Verlag, ISBN: 978-3540679219, Berlin.

Ogawa, H. (1992). Neural Networks Learning Generalization and Over-Learning, Proceeding of the International Conference on Intelligent Information Processing \& System, vol. 2, pp. $1-6$.

Ogawa, T.; Kanada, H. \& Kasano, H. (2003). Neural Network Localization of a Steel Ball in Impact Perforation Images, Proceeding of the SICE Annual Conference, pp.416-419. 
Ogawa, T.; Tanaka, S.; Kanada, H. \& Kasano, H. (2006). Impact Perforation Image Processing Using a Neural Network, Proceeeding of the SICE-ICASE International Joint Conference. pp. $3762-3765$.

Okubo, K. ; Ogawa, T. \& Kanada, H. (2007). Impact Perforation Image Processing Using a Self-Organizing Map, Proceeding of the SICE Annual Conference, pp.1099-1103.

Principe, J. C.; Euliano, N. R. \& Lefebvre, W. C. (2000). Neural and Adaptive Systems, John Wiley \& Sons, ISBN: 0-471-35167-9, New York.

Ripley, B. D. (2007). Pattern Recognition and Neural Networks, Cambridge University Press, ISBN: 978-0521717700, New York.

Zukas, J. A. (1990). High Velocity Impact Dynamics, John Wiley \& Sons, ISBN: 978-0471514442, New York. 


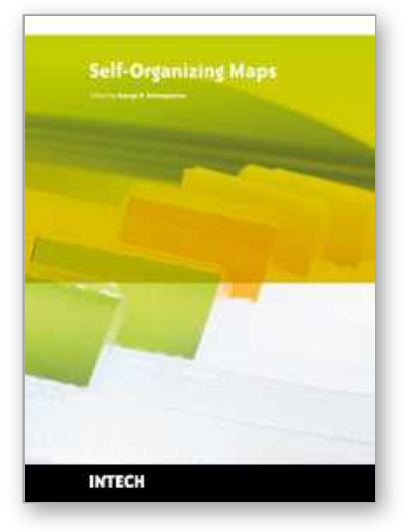

\author{
Self-Organizing Maps \\ Edited by George K Matsopoulos
}

ISBN 978-953-307-074-2

Hard cover, 430 pages

Publisher InTech

Published online 01, April, 2010

Published in print edition April, 2010

The Self-Organizing Map (SOM) is a neural network algorithm, which uses a competitive learning technique to train itself in an unsupervised manner. SOMs are different from other artificial neural networks in the sense that they use a neighborhood function to preserve the topological properties of the input space and they have been used to create an ordered representation of multi-dimensional data which simplifies complexity and reveals meaningful relationships. Prof. T. Kohonen in the early 1980s first established the relevant theory and explored possible applications of SOMs. Since then, a number of theoretical and practical applications of SOMs have been reported including clustering, prediction, data representation, classification, visualization, etc. This book was prompted by the desire to bring together some of the more recent theoretical and practical developments on SOMs and to provide the background for future developments in promising directions. The book comprises of 25 Chapters which can be categorized into three broad areas: methodology, visualization and practical applications.

\title{
How to reference
}

In order to correctly reference this scholarly work, feel free to copy and paste the following:

Takehiko Ogawa (2010). Image Clustering and Evaluation on Impact Perforation Test by Self-Organizing Map, Self-Organizing Maps, George K Matsopoulos (Ed.), ISBN: 978-953-307-074-2, InTech, Available from: http://www.intechopen.com/books/self-organizing-maps/image-clustering-and-evaluation-on-impactperforation-test-by-self-organizing-map

\section{INTECH}

open science | open minds

\section{InTech Europe}

University Campus STeP Ri

Slavka Krautzeka 83/A

51000 Rijeka, Croatia

Phone: +385 (51) 770447

Fax: +385 (51) 686166

www.intechopen.com

\section{InTech China}

Unit 405, Office Block, Hotel Equatorial Shanghai

No.65, Yan An Road (West), Shanghai, 200040, China

中国上海市延安西路65号上海国际贵都大饭店办公楼 405 单元

Phone: +86-21-62489820

Fax: $+86-21-62489821$ 
(C) 2010 The Author(s). Licensee IntechOpen. This chapter is distributed under the terms of the Creative Commons Attribution-NonCommercialShareAlike-3.0 License, which permits use, distribution and reproduction for non-commercial purposes, provided the original is properly cited and derivative works building on this content are distributed under the same license. 